\title{
De quelques savoirs langagiers nécessaires à l'exercice de la citoyenneté
}

\section{Bruno Maurer}

\section{(2) OpenEdition \\ 12 Journals}

Édition électronique

URL : http://journals.openedition.org/trema/1715

DOI : 10.4000/trema. 1715

ISSN : 2107-0997

\section{Éditeur}

Faculté d'Éducation de l'université de Montpellier

\section{Édition imprimée}

Date de publication : 1 octobre 1999

Pagination : 73-79

ISSN : 1167-315X

\section{Référence électronique}

Bruno Maurer, « De quelques savoirs langagiers nécessaires à l'exercice de la citoyenneté », Tréma [En ligne], 15-16 | 1999, mis en ligne le 01 octobre 1999, consulté le 01 mai 2019. URL : http:// journals.openedition.org/trema/1715 ; DOI : 10.4000/trema.1715

Ce document a été généré automatiquement le 1 mai 2019.

Trema 


\title{
De quelques savoirs langagiers nécessaires à l'exercice de la citoyenneté
}

\author{
Bruno Maurer
}

1 Pourquoi lier citoyenneté et savoirs langagiers, alors que la notion de citoyenneté est une notion à la frontière du juridique et du politique et que le rapport avec les activités de communication est rarement posé ? Il peut sembler y avoir là comme un paradoxe, mais ce paradoxe, nous tâcherons du moins de le démontrer, n'est qu'apparent. En effet, on peut commencer par rappeler que l'exercice de la citoyenneté suppose que le citoyen dispose d'une voix et qu'il soit capable de se faire entendre sur les sujets relevant de la vie de la cité. Mais ce n'est pas par cette entrée, somme toute traditionnelle et qui nous conduirait à faire l'inventaire de très hypothétiques minima langagiers permettant de participer au débat démocratique, que nous voudrions lier la question de l'exercice de la citoyenneté et la problématique linguistique. Nous nous placerons pour ce faire dans une optique moins juridique et politique, plus économique, de l'exercice de la citoyenneté.

\section{De la citoyenneté dans son rapport avec le langage}

2 Ainsi, pour répondre à cette question posée en guise de préambule, il nous faut poser quelques réflexions sur ce qu'est un citoyen, car beaucoup d'acceptions sont en concurrence. Etre citoyen, c'est, pour prendre une définition minimale, avoir un certain nombre de droits et de devoirs dans la cité. Qu'il soit d'abord clairement établi qu'en France, on est citoyen ou on ne l'est pas : il n'y a pas de degrés dans la citoyenneté. En revanche, une fois affirmé ce principe égalitariste, il n'en est pas moins indéniable que tous les citoyens n'exercent pas au même niveau leur citoyenneté, c'est-à-dire qu'ils n'ont pas tous la même capacité à faire valoir leurs droits politiques dans la cité et qu'ils n'ont pas non plus tous une égale conscience de leurs devoirs envers elle.

Or, pouvoir exercer pleinement sa citoyenneté, cela passe notamment, même si cela ne suffit pas, par l'aptitude à se mouvoir dans tous les milieux sociaux de la cité, à ne pas 
être enfermé seulement dans certaines sphères. La possibilité pour chacun d'effectuer ce que l'on appelle des trajets sociaux, de faire fonctionner à son profit l'ascenseur social fonde le pacte républicain sur lequel vit notre système politique: sans cela, ce pacte républicain risque de n'être qu'un marché de dupes. La possibilité de trajets sociaux en est le fondement, et elle en conditionne l'adhésion. Sans elle, on aboutit à une ghettoïsation, à une vie côte à côte de groupes sociaux différents, sans interaction réelle, à un éclatement du corps social, à une perte des valeurs communes, à une dissolution de l'unité, à une fracture sociale et à une fracture politique. Précisons au passage que cette analyse vaut pour le cas de la République française mais qu'elle ne correspondrait sans doute pas à d'autres modèles politiques et sociaux, anglo-saxons notamment.

Rejeter la tentation des ghettos.

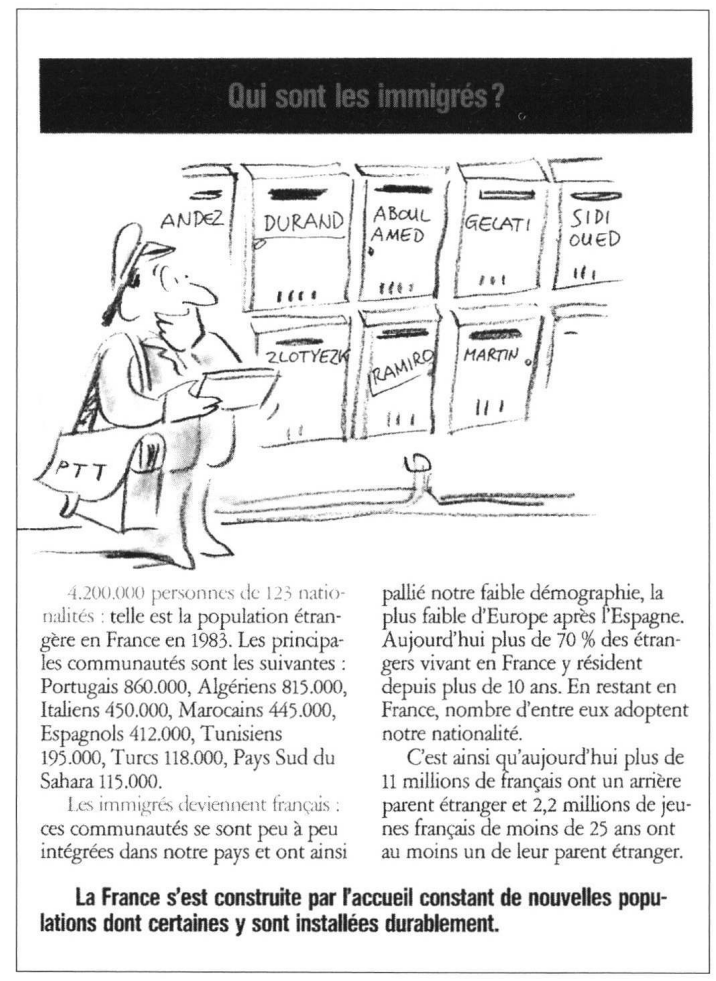

SECRÉTARIAT D'ÉTAT CHARGÉ DES IMMIGRÉS : Vivre ensemble : les immigrés parmi nous. Paris, Office National d'Immigration, 15 p., p. 6.

4 Cette aptitude à la mobilité sociale, qui est indissociablement liée à l'idée républicaine en France, suppose que les citoyens, égaux devant le langage, soient capables de communiquer partout et puissent, même en théorie, exercer leur action dans tous les milieux sociaux. De ce point de vue, un ensemble de compétences langagières semblent indispensables pour pouvoir exercer pleinement sa citoyenneté. On voit donc bien comment les deux termes, citoyenneté et savoirs langagiers, peuvent être liés, dès lors que l'on cesse de poser le problème de la citoyenneté de façon abstraite et que l'on envisage les comportements que l'exercice de celle-ci suppose. 


\section{Un handicap linguistique à compenser ou un autre rapport au langage à construire?}

Mais il ne faudrait pas réduire cette analyse au constat simple d'un déficit langagier de certains groupes sociaux, qui disposeraient de ressources linguistiques insuffisantes pour communiquer efficacement dans des situations variées, parce que cette affirmation ne reposerait sur aucune étude sérieuse et que l'hypothèse d'un tel handicap linguistique a toutes les chances de ne pas être fondée.

6 Plus que des savoirs langagiers concernant des moyens d'expression linguistique, il nous semble que ce qui devrait être au centre de la problématique c'est le développement d'une réflexion sur la valeur des formes en discours, leur portée dans l'interaction, la manière dont ces formes vont être perçues par le partenaire de l'interaction.

$7 \quad$ Que l'on nous permette d'illustrer la nécessité de ce type de sensibilisation par le récit d'une anecdote qui paraîtra caricaturale tant elle est révélatrice. Une émission quotidienne de France Inter, Le téléphone sonne, consacrée à la violence scolaire, à l'automne de 1996, proposait en guise d'ouverture et comme pour donner le ton aux débats l'interview d'une jeune collégienne en délicatesse avec l'école, qui décrivait ses rapports difficiles avec les enseignants. Elle confessait leur adresser, avec ses camarades, des gros mots, mais qu'elle caractérisait comme des "petits gros mots", sans réelle gravité. A une question d'un journaliste lui demandant "et c'est quoi ces petits gros mots?", elle répondit sur le ton de la minimisation : «ben je sais pas moi, enculé, bâtard quoi ».

Cette situation est très révélatrice des différences d'appréciation, et des difficultés de communication qui en résultent, qui peuvent exister entre le monde des adultes et celui des enfants : la jeune fille n'accorde visiblement pas la même valeur aux mots en question que l'enseignant qui va les recevoir et cette performance verbale va être, sinon le début d'un malentendu, du moins l'aggravation du fossé entre les deux personnes. La jeune fille en toute bonne foi ne maîtrise pas toutes les règles de la communication, la valeur des mots, celle qu'ils prennent en fonction de la situation de communication, en fonction de la personnalité de l'interlocuteur. Ce qui semble être en question, au fond, c'est sa maîtrise de la langue, mais non pas entendue comme un déficit de moyens linguistiques entendu en termes classiques de registres - mais comme un défaut d'analyse des paramètres de la situation de communication, comme un manque de réflexion sur la portée des mots utilisés. Sans en arriver à ces extrémités, combien de fois peut-on entendre des enseignants, dans les salles de professeurs des établissements du secondaire, se plaindre du comportement verbal de leurs élèves qui, sans être franchement incorrect, ne correspond pas aux marques de respect qu'en général les adultes attendent? L'analyse de la situation finit dans la plupart des cas par un constat désabusé : « on ne leur a pas appris la politesse à ces enfants ». De fait, on pourrait peutêtre commencer par les sensibiliser à la valeur des formes.

\section{De la citoyenneté à la civilité, matrice de la communication sociale}

Sur quels pôles axer cette réflexion de type métalinguistique? Comment l'orienter? Quels aspects de la communication orale faudrait-il mettre en avant? 
Avant de répondre précisément à cette question, disons que le principe général d'un tel programme pourrait être de parvenir à rendre explicites pour tous certaines conduites de communication dont les règles sont implicites et dont la maitrise fait défaut à bon nombre d'individus.

Les travaux des anthropologues et ethnologues de la communication montrent en effet que la construction de la compétence de communication requiert la maîtrise d'un ensemble de paramètres que nous ne percevons pas le plus souvent, immergés que nous sommes dans notre propre culture, mais dont nous faisons l'apprentissage de façon naturelle, par imitation des personnes que nous voyons communiquer dans notre entourage. Il en va ainsi pour ce qui est de l'ordre de la communication non-verbale, ainsi que pour tous les rituels qui gèrent nombre de situations de communication de la vie quotidienne.

11 Mais, si l'on se place dans une perspective d'apprentissage, tous les enfants ont-ils aujourd'hui la possibilité d'apprendre ces règles simplement par imprégnation, en suivant les modèles de communication qui leur sont proposés par leur entourage ? Tous les adultes ont-ils ce recul face aux enjeux du langage? Nous ne le pensons pas, compte tenu de plusieurs faits que nous nous contenterons de poser rapidement sans les élaborer :

- pluriculturalisme croissant des composantes de la société française, avec des pratiques de communication orales très variées, des modes de comportement langagier multiples;

- déficience des adultes, du fait des changements dans la structure de la famille, dans le domaine de la transmission des modes culturels et des valeurs;

- exposition par la télévision à des modes de communication souvent étrangers (américains) et représentant très fréquemment des rapports conflictuels entre les individus.

Parmi toutes ces règles, certaines nous semblent plus importantes stratégiquement que d'autres, si l'on se place du point de vue de la nécessité de tirer parti de ses interactions avec les autres, de la nécessité d'être reconnu par les autres comme un interlocuteur valable, acceptable, de la possibilité de communiquer de manière très large dans des milieux et des situations très variés. Il s'agit de celles qui concernent les phénomènes de négociation des faces dans l'interaction verbale, actes menaçant la face ou au contraire actes réparateurs, tels que les décrit $\mathrm{E}$. Goffman (1967).

Un exemple simple sera fourni par un acte tel « refuser une proposition ». Le même acte peut être réalisé par un francophone natif de bien des manières différentes et l'on s'accordera à penser que « ça va pas non ? » et « j'aurais été enchanté de pouvoir accepter mais je ne peux vraiment pas " représentent deux formulations qui se situent aux deux pôles d'un continuum de formes linguistiques ayant en commun de réaliser le même acte de parole. On peut analyser ces deux modes d'expression dans une perspective normative et dire que la première se situe dans un registre situé en dessous de la norme, alors que la seconde relève d'un registre soutenu. En réalité, la différence entre les deux formulations tient à un autre aspect, souvent négligé et totalement indépendant de la problématique de la norme et des registres: alors que "ça va pas non?» est une manière brutale, agressive peut-on dire, de refuser une proposition, l'autre phrase accomplit le même acte mais en ménageant la personne de l'interlocuteur. Or le fait qu'il y ait ou non agressivité n'est pas lié au choix du registre: un très soutenu «ta proposition est complètement absurde, comme à l'accoutumée du reste » sera au moins autant agressif que le familier «ça va pas non? ». En revanche, le « ça craint » des adolescents, pour familier qu'il soit, évite l'expression d'un jugement portant sur l'autre et est une façon peu agressive de 
refuser une proposition. Par delà les questions de registres, ces deux énoncés diffèrent radicalement par les intentions de communication dont ils sont porteurs : la première forme manifeste à l'égard de l'interlocuteur une intention que l'on pourrait qualifier de " conflictuelle», «agressive»; la seconde est au contraire "non conflictuelle», plus "consensuelle", ménageant la poursuite de l'interaction verbale en continuant à reconnaître l'autre comme un interlocuteur digne d'attention.

La position défendue ici est que nous ne sommes pas tous égaux devant la réalisation de tels actes de parole : certaines personnes savent les réaliser à la fois en consensus et en dissensus, parce qu'elles disposent dans leur environnement de modèles langagiers suffisamment divers pour se les approprier par imprégnation et également parce qu'elles savent mesurer les effets des formes linguistiques dans l'interaction verbale: pour cette dernière dimension, le rôle de l'entourage est également capital, qui peut, par des questions ou des remarques, aider au développement de cette conscience métalinguistique ; d'autres personnes, dans un environnement langagier moins riche, que ce soit au plan des moyens d'expression ou du point de vue du rapport au langage, dans un rapport moins réflexif, ont une maîtrise insuffisante du consensus et s'inscrivent le plus souvent en opposition forte à l'autre, tout simplement parce qu'ils n'ont pas le choix d'autres types de formulations ou parce qu'ils ne perçoivent pas suffisamment les différences du point de vue de leur portée. Les premiers auront toutes les chances de pouvoir tirer profit des interactions orales qu'ils auront avec les autres, dans des situations de communication variées, dans des sphères sociales diverses, les seconds risquent d'être enfermés dans des sphères de communication de connivence, et être, hors de ces sphères, les objets de jugements extrêmement négatifs, pouvant aller jusqu'à des jugements d'exclusion. On sait que la maîtrise des compétences de communication orale est un type de maîtrise de plus en plus important dans les sociétés modernes et que c'est sur cette base que les individus sont de plus en plus souvent évalués. Les premiers pourront plus facilement que les seconds faire un plein usage de leur citoyenneté, parce qu'ils seront reconnus par leurs interlocuteurs comme des partenaires possibles; les seconds seront toujours au risque de la marginalisation, prisonniers d'un langage qu'ils ne maîtrisent pas assez pour en tirer un profit personnel.

\section{De nouvelles pistes pour une didactique de l'oral}

On voit donc bien quels peuvent être les enjeux d'une didactique de l'oral qui viserait au développement de compétences de communication relevant de la conversation ordinaire et pas seulement de l'exercice de types de discours oraux ritualisés. L'objectif concret d'une didactique de l'oral partant de ces réflexions pourraient être de diversifier les moyens linguistiques dont disposent les locuteurs pour réaliser des actes de parole $\mathrm{du}$ quotidien qui sont souvent très difficiles à négocier car chargés d'enjeux, et de commencer à le faire par le biais d'un enseignement à l'école dès le niveau primaire, afin que tous les élèves aient à leur disposition le plus large éventail possible de ressources. Il faut, dans le même temps, développer une réflexion autour de ces formes linguistiques et des intentions de communication qu'elles véhiculent, de manière à ce que les locuteurs puissent effectuer clairement des choix conscients et puissent ajuster au mieux le mode d'expression choisi à l'intention de communication voulue. La problématique trop strictement normative des registres («bien» parler / «mal» parler), rapportée à un modèle idéal qui est en fait une norme strictement sociale, pourrait être dépassée au 
profit d'une réflexion sur la manière de gérer harmonieusement ou conflictuellement la communication.

Mais, en même temps, il serait illusoire bien sûr de penser que l'on résoudra tous les problèmes de citoyenneté par le biais d'une intervention didactique sur les compétences orales et nous n'avons pas pareille prétention; simplement, nous pouvons peut-être apporter une pierre à la construction de cette citoyenneté qui passe de plus en plus par la communication, dont on sait, depuis les travaux de Brown et Levinson (1987), qu'elle n'est possible que par l'observation de rituels de politesse, qui ne sont pas simplement de la " civilité » mais qui sont au fondement même des relations sociales.

Parallèlement, la réflexion que l'on pourrait, par de telles orientations didactiques, développer chez les élèves pourrait avoir un impact plus direct sur les comportements violents. Il s'agit peut-être même là d'un de ses objectifs les plus immédiats. En effet, une part importante de ce que l'on dénonce comme étant de la "violence ", à l'école ou dans la rue, est souvent de la violence verbale. Et quand on prend le temps d'interroger un élève sur les raisons qui l'ont poussé à agresser verbalement un enseignant ou un camarade, on s'aperçoit que l'enfant n'avait pas toujours l'intention d'être agressif et qu'il ne donnait pas à la forme linguistique employée la même valeur que celle que lui a conférée celui qui en était le destinataire. C'est peut-être là une autre conception de la citoyenneté qui peut se trouver confortée, celle qui tend à substituer à la violence des relations le cadre de la négociation, l'espace de l'échange des représentations, quand bien même celles-ci seraient discordantes.

\section{BIBLIOGRAPHIE}

BROWN P \& LEVINSON S.C. : Politeness : some universals in language usage. Cambridge, Cambridge University Press, 1987.

GOFFMAN E. : Ritual interaction. Essay on Face-to-face behaviour. New York, Doubleday, 1967.

(Trad. fr. : Les Rites d'interaction. Paris, Ed. de Minuit, 1974).

\section{RÉSUMÉS}

A partir de réflexions de type socio-économiques sur l'exercice de la citoyenneté, il apparaît important de développer chez tous les citoyens des compétences de communication permettant de se faire reconnaître par autrui comme un interlocuteur valable. C'est à ce prix qu'une réelle mobilité sociale sera possible, sur laquelle se fonde l'idée même de pacte républicain en France. C'est sur le développement d'aptitudes orales de communication que cette contribution met l'accent, notamment sur la nécessaire prise de conscience des valeurs pragmatiques des formes linguistiques, leur rôle dans la gestion des interactions verbales.

On the basis of reflections of a socio-economic nature on the actual exercise of citizenship, it appears important to develop in all citizens such communication skills as others may acknowledge them in a positive fashion. It is on such a basis that genuine upward mobility may 
become possible, this upward mobility being the very cornerstone of the Social Contract between the state and the people in France. This research paper focuses on the development of oral aptitudes of communication, particularly on the indispensible development of an awareness of the pragmatic values of linguistic forms and of their role in oral interactions management.

INDEX

Keywords : citizenship, Communication, development, metalinguistic aptitude, Pacte Républicain (Social Contract between the state and the people), skill, speech interaction

Mots-clés : acte de parole, aptitude métalinguistique, citoyenneté, communication, compétence, développement, pacte républicain

\section{AUTEUR}

\section{BRUNO MAURER}

IUFM de Montpellier et Université de Montpellier III, équipe Didaxis 